\title{
A self paced treadmill walking test for breathless patients
}

\author{
A BEAUMONT, A COCKCROFT, A GUZ \\ From the Department of Medicine, Charing Cross and Westminster Medical School, London
}

ABSTRACT A treadmill exercise test is described that retains the advantages of self paced walking but allows the measurement of cardiorespiratory variables. A horizontal treadmill was modified to allow patients to control their own speed. During exercise continuous measurements of speed, distance, heart rate, arterial oxygen saturation, and stride length were made and subjective assessments of breathlessness were recorded on a visual analogue scale. Ten men with severe chronic obstructive lung disease performed a 12 minute corridor walk and a self paced 12 minute treadmill walk on the same day and repeat treadmill walks on different days. Six of them performed three walks or more. Six patients had ventilatory measurements during treadmill exercise. There was no significant difference $(p>0.1)$ in the distance covered during corridor and treadmill walks, and distances on the treadmill were repeatable after the first walk. Use of a mouthpiece significantly reduced the distance covered on the treadmill. The self paced treadmill walk is a simple repeatable test and has advantages over both a corridor walking test and standard progressive tests for assessment of breathless patients.

A 12 minute exercise test was originally described by Cooper ${ }^{1}$ as an indication of physical fitness in young normal subjects. He found a close correlation between the distance run in 12 minutes and the maximum oxygen uptake measured on a treadmill. McGavin and coworkers ${ }^{2}$ modified the 12 minute exercise test for patients with chronic obstructive lung disease. They asked patients to cover as much distance as they could in 12 minutes along a level hospital corridor and they found this to be a reproducible index of exercise tolerance. ${ }^{2}$ The reproducibility of the 12 minute walking distance has been confirmed by others. ${ }^{34}$

The major disadvantage of this type of corridor walking test is the lack of information about physiological and symptomatic changes during exercise. Monitoring these changes is important when studying mechanisms of exercise limitation and when investigating the effects of exercise training programmes in disabled patients.

Our study was undertaken to develop and validate a self paced treadmill walking test, which was similar

Address for reprint requests: Dr A Cockcroft, Department of Medicine, Charing Cross and Westminster Medical School, London W6 8RF.

Accepted 2 January 1985 to the 12 minute corridor walking test in that patients were in complete control of their speed, but which allowed measurements of physiological variables during the exercise. The treadmill walk was validated in two ways: by comparison with the results of a corridor walk performed on the same day and by comparison of the results of repeated treadmill walks on different days. The work has already been presented in preliminary form. ${ }^{5}$

\section{Methods}

\section{SUBJECTS}

We studied 10 men aged $46-73$ years. All had chronic airways obstruction, with mean forced expiratory volume in one second $\left(\mathrm{FEV}_{1}\right)$ of 1.03 (SD 0.27, range 0.54-1.39) 1 and mean forced vital capacity (FVC) of 2.68 (SD 0.60, range 1.6-3.74)l. Two women were also tested in the comparison of treadmill walks with and without a mouthpiece $\left(\mathrm{FEV}_{1}\right.$ 0.8, FVC 2.3; FEV 1 0.6, FVC 2.3 l).

\section{PROTOCOL}

Spirometry was performed in all patients on the days of exercise testing. The 10 men performed a standardised 12 minute corridor walk along a level hospital corridor and a treadmill walk on the same day, 
in random order. A minimum of 45 minutes' rest was allowed between the two exercises. All 10 patients completed a second treadmill walk on a different day and six of them performed a third treadmill walk. Six patients (four of the men and the two women) had ventilatory measurements made during the treadmill walk with a mouthpiece and pneumotachograph and this walk was compared with a control treadmill walk performed on the same day.

\section{THE CORRIDOR WALK}

The corridor walk was carried out as described by McGavin et al. ${ }^{2}$ The patients were instructed to walk as far as they could in 12 minutes; they were told to keep going if possible but they could slow down or even stop if necessary. An operator accompanied each patient to record distance and time, and to give encouragement with standard phrases. The number of right heel strikes over $40 \mathrm{~m}$ distances was recorded for calculation of stride length.

\section{THE TREADMILL WALK}

The treadmill walk was performed on a horizontal treadmill (Marquette Electronics Ltd, USA) fitted with a remote control module modified for the patient's use. This comprised four easily accessible push buttons that allowed the treadmill to be stopped, started, speeded up, or slowed down. Stopping and changing speed occurred gradually, with acceleration or deceleration of $100 \mathrm{~m} \mathrm{~min}^{-2}$; starting, although abrupt, was always from the slowest speed $(1.5 \mathrm{~km} / \mathrm{h})$ and with the operator's assistance. Patients practised walking on the treadmill before starting the 12 minute walk. Standardised instructions and encouragement similar to those for the corridor walk were given during the exercise via a tape recording. Patients were instructed to walk "as far as possible" during the time-that is, as fast as possible. They were told that they could slow down or even stop if necessary. During the test the voltage output of speed from the treadmill was fed into a Mini analogue computer (AEI, USA), which integrated the signal to give a voltage output of distance.

Stride length was derived by the analogue computer: the number of right heel strikes recorded by a pedometer was divided into distance covered every 30 seconds. The pedometer consisted of a calculator keyboard switch taped into the right shoe under the heel. These signals were recorded continuously on a Mingograf strip chart recorder (Siemens, Holland). Arterial oxygen saturation was monitored at rest and throughout the exercise with a spectrophotometric ear oximeter ${ }^{6}$ (Hewlett Packard, USA). Heart rate and electrocardiogram were monitored throughout.
Subjective assessments of breathlessness were recorded during the treadmill walk on a visual analogue scale. ${ }^{7}$ The patients were presented with a $10 \mathrm{~cm}$ visual display on which a red light could be positioned by a linear sliding potentiometer. The left end of the scale was labelled "not at all breathless" and the right end "extremely breathless." Patients were asked to signal at the moment when they first felt breathless and then to indicate how breathless they felt on the visual analogue scale every $30 \mathrm{sec}-$ onds, prompted by a light signal. A mean breathlessness score was obtained by summing all the scores during the test and dividing by 24 (the number of half minute periods); this has been used as an index of overall breathlessness.

The six patients in whom ventilatory measurements were made during the treadmill walk breathed through a pneumotachograph (Fleisch No 1 or 2) attached to a Fenyves and Gut Ergostar ${ }^{8}$ (Basel, Switzerland). Mixed expired gas was analysed for carbon dioxide and oxygen; and carbon dioxide production, oxygen consumption, and ventilation were calculated over half minute periods. All equipment was calibrated before every exercise test.

\section{ANALYSIS}

Corridor and treadmill walking distances were compared by means of a paired $t$ test. Repeatability of the treadmill walk was assessed by comparing distances covered in each quarter of the time for separate walks; paired $t$ tests were used to compare three, six, nine, and 12 minute distances for walks 1 and 2 , and 2 and 3 .

\section{Results}

Most patients found the treadmill walk acceptable and had no difficulty controlling treadmill speed. Training patients to walk normally on the treadmill was accomplished fairly easily. All patients but one experienced some reduction in oxygen saturation during the treadmill exercise, with a maximum fall of $18 \%$ (from $92 \%$ to $74 \%$ ). No patient complained of chest pain during the tests but ST segment depression consistent with ischaemic heart disease was noted in one patient. Figure 1 is shown as an example of the sort of information that can be obtained during a treadmill walk. In this particular patient increases in speed were accompanied by increases in the visual analogue scale score for breathlessness at a constant level of oxygen saturation.

\section{COMPARISON OF TREADMILL AND CORRIDOR}

WALKS

The 10 men who performed both corridor and 
treadmill walks said that walking on the treadmill was almost as easy as walking in the corridor. The relationship of stride length to walking speed seemed similar for corridor and treadmill walks (fig $2)$. The distance walked on the treadmill was not significantly different $(p>0.1)$ from that covered in the corridor on the same day by the group as a whole
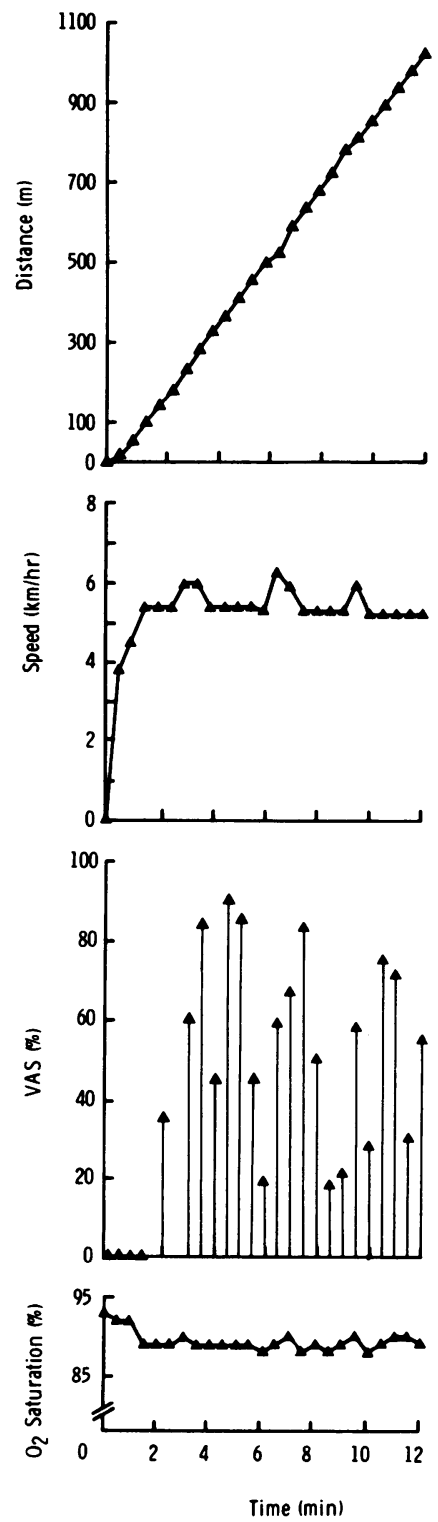

Fig 1 A selfpaced 12 minute treadmill walk with continuous measurements of distance, speed, and arterial oxygen saturation. Subjective breathlessness was scored on a visual analogue scale (VAS). (fig 3). Two patients stopped walking during one or both of the tests; both stopped more frequently in the corridor.

\section{REPEATABILITY OF TREA DMILL WALKS}

The distance covered during the first treadmill walk was significantly shorter than that covered during the second $(n=10, p<0.05$ : fig 4$)$. For the patients who completed three or more treadmill walks, there was no significant difference between the second and third walking distances $(n=6, p>0.1$, fig 5); in this subgroup there was also no significant difference between the first and second walking distances $(p>$ 0.1 ), and this presumably reflects the reduced size of the subgroup. Despite the greater distance (and speed) of the second treadmill walk, the mean score for breathlessness assessed by visual analogue scale was not different from that recorded during the first treadmill walk $(p>0.2)$. Similarly, breathlessness scores did not differ between the second the third treadmill walks $(p>0.4)$.

Patients differed in the way they achieved their total 12 minute distance on the treadmill: some walked at a steady pace throughout, while others varied their speed during the test. For individual patients the pattern was relatively constant: when the second and third treadmill walks were compared, the distances covered over consecutive three minute periods of the tests did not differ significantly between tests (six men).

\section{EFFECT OF THE MOUTHPIECE AND PNEUMOTACHOGRAPH}

Treadmill walking distance was less for five out of six patients when they performed the test with a mouthpiece and heated pneumotachograph (Fleisch No 2-dead space $55 \mathrm{ml}$ ) than during a control walk (fig 6). The difference in distance covered was significant $(\mathrm{p}<0.05)$. Mean visual analogue scale scores for the control walk (mean 25.8 (SD 24.5)) and the walk with a mouthpiece (mean $27.8(23.8)$ ) were not significantly different $(p>0.4)$. Patients reported that the mouthpiece made the treadmill walk more unpleasant.

\section{Discussion}

These results suggest that the 12 minute self paced treadmill walk can be used for physiological evaluation during exercise of patients disabled by breathlessness. The similar distances covered by patients on the treadmill and in the corridor, and the fact that the relation of stride length and speed was apparently the same for the two types of walk, suggests that patients were walking on the treadmill in the same way as in the corridor. 
The treadmill walking distance was repeatable after the first walk. We now use the first treadmill walk as a training procedure, in common with other investigators who have used the corridor walk. Not only was the total distance repeatable after the first treadmill walk but intermediate three minute distances were remarkably consistent for individual subjects, suggesting that each had their own "best way" of covering the greatest distance in the time. Interestingly, the mean breathlessnes score for the second treadmill walk was the same as for the first, despite the greater distance on the second occasion. This could mean that the patients were limiting themselves at the same symptomatic level on the two occasions; the lesser breathlessness relative to speed on the second occasion is unexplained.

The treadmill walking test allows measurements of gas exchange to be made during exercise, which may be important in studies of the mechanisms that limit exercise tolerance in patients with chronic obstructive lung disease. This, however, requires the patient to breathe through a mouthpiece and pneumotachograph, which we found significantly reduced the distance walked. Visual analogue scale scores were not reduced despite the shorter distance covered when a mouthpiece was used, again suggesting that patients were limiting themselves at a given level of breathlessness. In view of this effect of the mouthpiece and pneumotachograph, it is clearly reasonable to compare walking distances on the treadmill only if they are performed in similar cir- cumstances (that is, with or without a mouthpiece) in studies of the effects of, for example, exercise training. Other variables that are difficult to measure during corridor walking but can easily be meas-

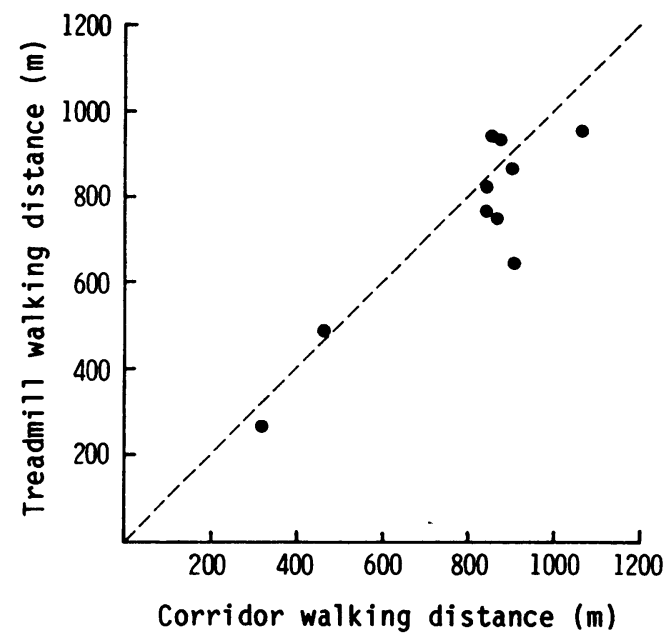

Fig 3 Comparison of 12 minute corridor walking distance for 10 patients with self paced 12 minute treadmill walking distance performed on the same day, in random order. Mean treadmill distance $=742(S D 224) \mathrm{m}$; mean corridor distance $=791$ (224) $\mathrm{m} ; S D$ of difference $=100 \mathrm{~m}(\mathrm{p}>$ $0.1) ; r=0.898$, slope $=0.895$, intercept $=-33.4 \mathrm{~m}$. The dashed line is the line of identity.

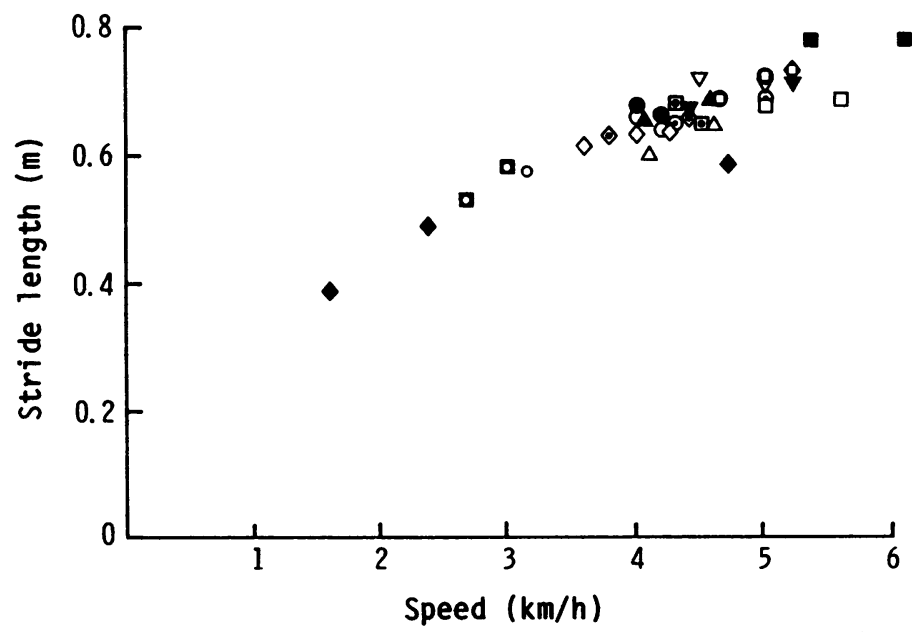

Fig 2 Relationship between average stride length and speed for corridor (over $40 \mathrm{~m}$ distance) and treadmill (over 30 second period) walking; pauses are not included. Each of the 10 patients is represented by a different symbol; open symbols are for corridor measurements, closed symbols for the treadmill. For some patients more than one point of comparison between stride length and speed was possible for either treadmill or corridor walking and all points are shown. 
ured during the treadmill walk include ECG configuration, assessments of breathlessness on a visual analogue scale during the exercise, and arterial oxygen saturation measured by ear oximeter. It is even possible to sample from an arterial line during the treadmill walk.

This type of exercise test may have advantages

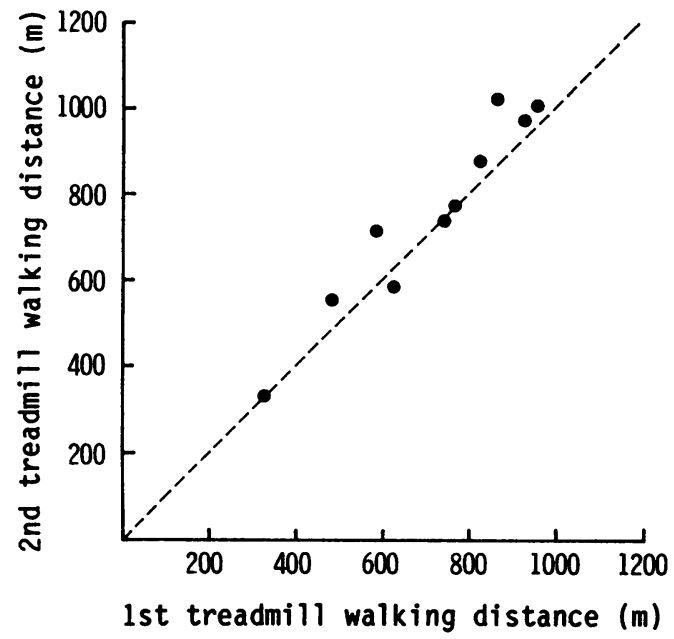

Fig 4 Comparison of distance walked on the treadmill on the first and second occasions for 10 patients. First walk: mean distance $=712(S D 203) \mathrm{m}$; second walk: mean distance $=758(221) \mathrm{m} ; S D$ of difference $=58 \mathrm{~m}(p<$ $0.05) ; r=0.964$, slope $=1.051$ intercept $=11.1 \mathrm{~m}$. The dashed line is the line of identity.

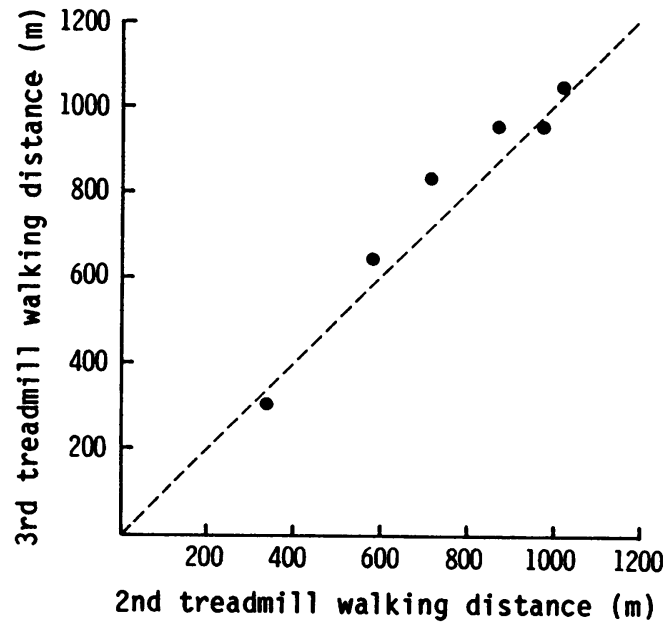

Fig 5 Comparison of distance walked on the treadmill on the second and third occasions for six patients. Second walk: mean distance $=751$ (SD 261) $\mathrm{m}$; third walk: mean distance $=788$ (273) $\mathrm{m} ; S D$ of difference $=56 \mathrm{~m}$ $(p>0.1) ; r=0.979$, slope $=1.027$, intercept $=17.6 \mathrm{~m}$. The dashed line is the line of identity.

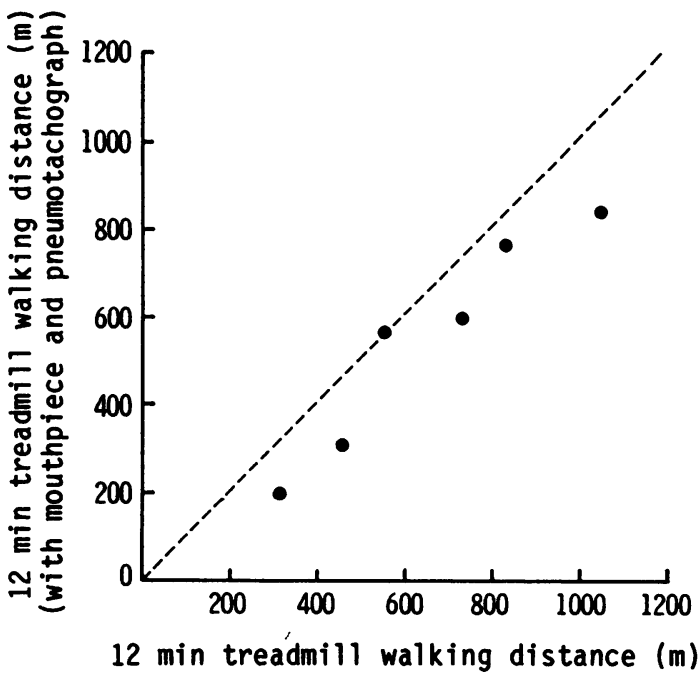

Fig 6 Effect of mouthpiece and pneumotachograph on 12 minute treadmill walking distance for six patients. Control walk: mean distance $=658$ (SD 273) m; " mouthpiece walk" mean distance $=547(251) \mathrm{m} ; \mathrm{SD}$ of difference $=117 \mathrm{~m}(p<0.05) ; r=0.960$, slope $=0.885$, intercept $=-35.2 \mathrm{~m}$. The dashed line is the line of identity.

over the standard progressive respiratory exercise test, ${ }^{10}$ on bicycle or treadmill in very disabled patients. Such patients find progressive tests frightening and unpleasant and often can exercise only for very short periods, producing limited data for analysis. More information is available from a longer, self paced test. Measurements at the time of stopping and restarting exercise, or with voluntary changes in speed, may be of particular value in assessing factors limiting everyday activity. This information is not obtained during a progressive test. Thus a self paced treadmill walk may be better than either a corridor walking test or a standard progressive test for assessing severely breathless patients. So far we have formally assessed the test only for patients disabled by respiratory disease but preliminary work suggests that it will also be useful in the assessment of patients disabled by breathlessness and fatigue due to poor left ventricular function.

We thank Dr Lewis Adams for much helpful discussion.

\section{References}

1 Cooper KH. A means of assessing maximal oxygen intake. JAMA 1968;203:201-4.

2 McGavin CR, Gupta SP, McHardy GJR. Twelve 
minute walking test for assessing disability in chronic bronchitics. Br Med J 1976;i:822-3.

3 Mungall IPF, Hainsworth R. Assessment of respiratory function in patients with chronic obstructive airways disease. Thorax 1979;34:254-8.

4 O'Reilly JF, Shaylor JM, Fromings KM, Harrison BDW. The use of the 12 minute walking test in assessing the effect of oral steroid therapy in patients with chronic airways obstruction. $\mathrm{Br} J$ Dis Chest 1982; 76:374-82.

5 Beaumont A, Cockcroft A, Guz A. A treadmill walking test for breathless patients. Clin Sci 1983;64:11P (abstract).

6 Saunders NA, Powles ACP, Rebuck AS. Ear oximetry: Accuracy and practicability in the assessment of arter- ial oxygenation. Am Rev Respir Dis 1976;113:745-9.

7 Adams L, Chronos N, Lane R, Guz A. The measurement of breathlessness induced in normal subjects: validity of two scaling techniques. Clin Sci 1985;69: 7-16.

8 Reinhard U, Muller PH, Schmulling RM. Determination of anaerobic threshold by the ventilation equivalent in normal individuals. Respiration 1979;38:36-42.

9 Cockcroft A, Beaumont AG, Adams L, Guz A. Arterial oxygen desaturation during treadmill and bicycle exercise in patients with chronic obstructive airways disease. Clin Sci 1985;68:327-32.

10 Jones NL, Campbell EJM. Clinical exercise testing. 2nd ed. Philadelphia: WB Saunders, 1982. 